\title{
The Influence of Non-Linear Frequency Compression on the Perception of Speech and Music in Patients with High Frequency Hearing Loss
}

\author{
Jungmin Ahn ${ }^{1}$, Ji Eun Choi ${ }^{2}$, Ju Yong Kang ${ }^{1}$, Ik Joon Choi ${ }^{1}$, Myung-Chul Lee ${ }^{1}$, \\ Byeong-Cheol Lee ${ }^{1}$, Sung Hwa Hong ${ }^{3}$, and II Joon Moon ${ }^{4}$ \\ ${ }^{1}$ Department of Otorhinolaryngology-Head and Neck Surgery, Korea Cancer Center Hospital, Seoul, Korea \\ ${ }^{2}$ Department of Otorhinolaryngology-Head and Neck Surgery, Dankook University Hospital, Cheonan, Korea \\ ${ }^{3}$ Department of Otorhinolaryngology-Head and Neck Surgery, Samsung Changwon Hospital, \\ Sungkyunkwan University School of Medicine, Seoul, Korea \\ ${ }^{4}$ Department of Otorhinolaryngology-Head and Neck Surgery, Samsung Medical Center, Sungkyunkwan University School of Medicine, \\ Seoul, Korea
}

\author{
Received June 9, 2020 \\ Revised September 16, 2020 \\ Accepted November 10, 2020 \\ Address for correspondence \\ Il Joon Moon, MD, PhD \\ Department of Otorhinolaryngology- \\ Head and Neck Surgery, \\ Sungkyunkwan University \\ School of Medicine, \\ Samsung Medical Center, \\ 81 Irwon-ro, Gangnam-gu, \\ Seoul 06351, Korea \\ Tel +82-2-3410-3579 \\ Fax +82-2-3410-6987 \\ E-mail moonij@skku.edu
}

Background and Objectives: Non-linear frequency compression (NLFC) technology compresses and shifts higher frequencies into a lower frequency area that has better residual hearing. Because consonants are uttered in the high-frequency area, NLFC could provide better speech understanding. The aim of this study was to investigate the clinical effectiveness of NLFC technology on the perception of speech and music in patients with high-frequency hearing loss. Subjects and Methods: Twelve participants with high-frequency hearing loss were tested in a counter-balanced order, and had two weeks of daily experience with NLFC set on/off prior to testing. Performance was repeatedly evaluated with consonant tests in quiet and noise environments, speech perception in noise, music perception and acceptableness of sound quality rating tasks. Additionally, two questionnaires (the Abbreviated Profile of Hearing Aid Benefit and the Korean version of the International Outcome Inventory-Hearing Aids) were administered. Results: Consonant and speech perception improved with hearing aids (NLFC on/off conditions), but there was no significant difference between NLFC on and off states. Music perception performances revealed no notable difference among unaided and NLFC on and off states. The benefits and satisfaction ratings between NLFC on and off conditions were also not significantly different, based on questionnaires, however great individual variability preferences were noted. Conclusions: Speech perception as well as music perception both in quiet and noise environments was similar between NLFC on and off states, indicating that real world benefits from NLFC technology may be limited in Korean adult hearing aid users.

J Audiol Otol 2021;25(2):80-88

KEY WORDS: Hearing loss, High frequency · Hearing aids · Speech perception · Music · Sound.

\section{Introduction}

Many patients with sensorineural hearing loss have a sloping high-frequency loss with relatively good threshold level

This is an Open Access article distributed under the terms of the Creative Commons Attribution Non-Commercial License (https://creativecommons.org/licenses/by-nc/4.0/) which permits unrestricted non-commercial use, distribution, and reproduction in any medium, provided the original work is properly cited. at low frequencies. Based on data from the hierarchical clustering method of the 2009-2012 Korean National Health and Nutrition Examination Survey, among eleven hearing-impaired audiograms (27.89\%), 7 (17.81\%) were flat-type and 4 (9.08\%) were sloping-type [1]. These listeners with sloping loss often miss out on high-frequency components of speech, such as consonant sounds, and can have difficulties in understanding speech in a noisy background. Many sounds or phonemes that 
contribute significantly to speech recognition primarily include high-frequency components. For example, recognizing important grammar markers such as /s/ and /z/ in English relies heavily on access to high frequencies [2].

Restoring high-frequency audibility has always been an important goal of hearing-aid design, since most listeners with hearing loss have poor audibility in the high-frequency regions. However, simple amplification of the high-frequency components does not improve speech intelligibility without acoustic feedback. In addition to difficulties with obtaining sufficient audibility, high-frequency components of the speech spectrum could not be audible because of the presence of nonfunctioning inner hair cells of the cochlea (cochlear dead regions, CDRs) [3].

One type of strategy is a frequency lowering technology that shifts the high-frequency information to a lower-frequency area, making the sounds fall into the limited processing bandwidth of the hearing device and the physiological audible bandwidth. There are several types of frequency lowering technologies. Of them, non-linear frequency compression (NLFC) is a way of reducing the frequency bandwidth of the output relative to the input through compression. In NLFC technology, the high-frequency band is compressed by a predetermined compression ratio (CR), while the frequency component of the low frequency band remains unprocessed. This technique can be particularly useful for consonant perception. However, previous studies have shown inconsistent results for the effects of NLFC on consonant perception in hearing-impaired listeners. Several studies have reported that improved perception of consonants can be achieved by activating NLFC [4-6]. Glista, et al. [7] tested phoneme perception with NLFC and conventional processing (CP) in 24 hearing-impaired participants with sloping hearing loss. The phoneme perception tasks included speech detection thresholds of /s/ and /sh/, consonant recognition, plural recognition, and vowel recognition. For consonant recognition, 10 high-frequency consonant, $/ \mathrm{t}$, d, $\mathrm{f}, \mathrm{j}, \mathrm{k}, \mathrm{s}, \int, \mathrm{t}, \mathrm{\partial}, \mathrm{z} /$, were used. The results suggested that speech detection thresholds of /s/ and /sh/, as well as high frequency consonants and plural recognition scores, showed a more remarkable improvement with NLFC than without it. In addition, Wolfe, et al. [4] reported that NLFC provided a more notable improvement in the recognition rate of some high frequency consonants (including /s/ and /sh/) after a six-week adaptation period for children with moderate hearing loss. With the effect of long-term use, after 6 months of use, the perception of / $t$ / was significantly better when NLFC was on than when it was off. However, a few other studies have demonstrated negative results in consonant perception [8-10]. Simpson, et al. [10] recruited 7 participants with steeply sloping hearing loss and none of them showed significant differences in consonant recognition between NLFC and CP. In addition, Perreau, et al. [8] recruited 17 adults with moderate or moderateto-severe hearing loss and tested their consonant perception in quiet condition by using an Iowa Consonant Recognition test. The results revealed that there was no significant difference between NLFC and CP in consonant recognition.

There is little research on the influence of NLFC on music perception and sound quality. For music perception, Uys, et al. [11] reported that NLFC significantly improves perception of timbre and melody. In addition, the activation of NLFC also improved the music qualities of overall fidelity, tininess, and reverberance. Parsa, et al. [12] also investigated whether frequency compression parameters affect the sound quality of music. The results were not significantly deteriorated unless the NLFC setting was too strong. For sound quality, Bohnert, et al. [13] recruited 11 hearing impaired adults, and asked them to complete a questionnaire for obtaining of subjective data of general benefit and sound quality of NLFC technique. The results showed that NLFC increased satisfaction of sound quality in 8 out of 11 . However, some studies showed no improvement in sound quality associated with NLFC [10,14].

Although NLFC is a technically innovative scheme, the inconsistent results on the efficacy of NLFC might be related to the variety of experimental designs employed. In addition, little research has been done for the effects of NLFC technology in languages other than English. It is well known that audibility of high-frequency linguistic information is imperative for optimal recognition of speech sound. The high-frequency phoneme $/ \mathrm{s} /$ is estimated to be the third most frequent phoneme in the English language, and it provides grammatical clues such as passive, plural and verb tenses. Therefore, NLFC technique was emphasized for English speaking hearing aid uses. However, since the frequency bands and features of speech spectrums between Korean and English are different, the effect of NLFC may be different in hearing aid users who speak and listen to Korean. Thus, the aim of this study was to investigate the effect of activating NLFC on the perception of speech and music in Korean adult hearing aid users with high-frequency hearing loss.

\section{Subjects and Methods}

\section{Patients}

A total of twelve participants (mean age 52.8 years; range 20 years to 81 years; standard deviation $=23.9$ years) with highfrequency hearing loss were enrolled. All participants had no self-disclosed history of neurological or cognitive impairment. The audiometric data is shown in Fig. 1A. All participants had 
a symmetrical audiogram with a slope of $5 \mathrm{~dB} /$ octave at frequencies above $1 \mathrm{kHz}$. The average pure-tone threshold at high frequencies $(4,6$, and $8 \mathrm{kHz})$ was $86.95 \pm 14.09 \mathrm{~dB}$, and the average difference in hearing threshold between $1 \mathrm{kHz}$ and $8 \mathrm{kHz}$ was $41.25 \pm 18.23 \mathrm{~dB}$.

Demographic information for each participant is shown in Table 1. The participants consisted of ten males and two females, all of whom were native Korean speakers. Eight of them had used binaural hearing aids, and the average dura- tion of hearing aid use was $75.83 \pm 55.89$ months. No participants had reported prior experience with frequency lowering technology.

All participants were fitted unilaterally with receiver-in-the ear (RIE) type devices. Two manufacturer hearing aids were used in this study: Audéo S smart V (Phonak, Stäfa, Switzerland) and LiNX2 RIE 62 (GN ReSound, Ballerup, Denmark). Hearing aid fitting was performed by experienced audiologists using the NAL-NL2 formula. The NLFC algorithm was
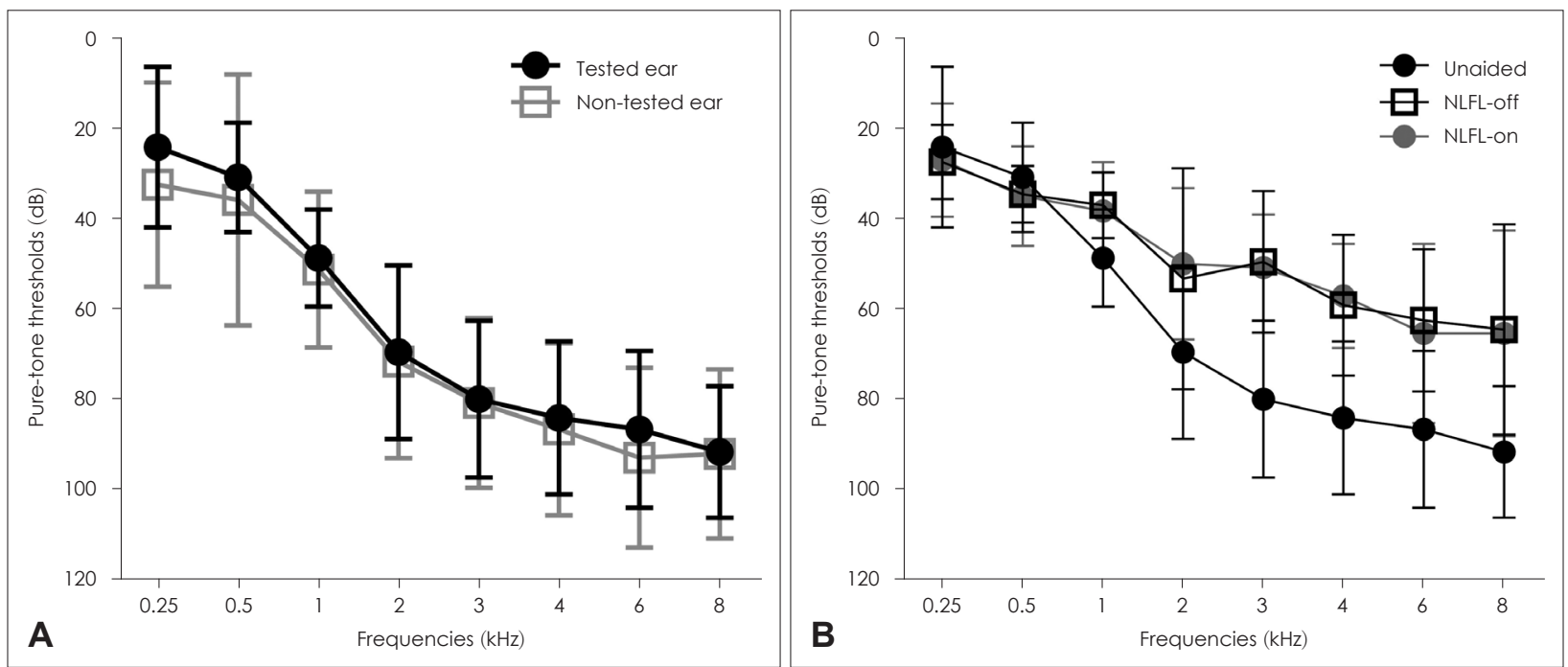

Fig. 1. Audiogram for participants. A: Average pure tone thresholds for tested and non-tested ears. All participants had an audiogram with a slope of $5 \mathrm{~dB} /$ octave at frequencies above $1 \mathrm{kHz}$, and had symmetrical hearing loss. $\mathrm{B}$ : The pure tone threshold at each frequency for the three listening conditions (unaided, with NLFC-off, and with NLFC-on). There was a significant difference in pure-tone thresholds between unaided and aided conditions at frequencies above $1 \mathrm{kHz}$ (all $p<0.001$ ). However, the pure-tone threshold was similar between the two aided listening conditions (NLFC-on and -off). NLFC: non-linear frequency compression.

Table 1. Demographic features of enrolled participants

\begin{tabular}{|c|c|c|c|c|c|c|c|c|c|c|c|c|c|c|}
\hline \multirow{2}{*}{ Sex } & \multirow{2}{*}{ Age } & \multirow{2}{*}{$\begin{array}{c}\text { Tested } \\
\text { ear }\end{array}$} & \multicolumn{9}{|c|}{ Hearing threshold (dB) } & \multirow{2}{*}{$\begin{array}{l}\text { Duration } \\
\text { of HA use } \\
\text { (months) }\end{array}$} & \multirow{2}{*}{$\begin{array}{l}\text { Cut-off } \\
\text { frequency } \\
\quad(\mathrm{kHz})\end{array}$} & \multirow{2}{*}{$C R$} \\
\hline & & & $250 \mathrm{~Hz}$ & $500 \mathrm{~Hz}$ & $750 \mathrm{~Hz}$ & $1 \mathrm{kHz}$ & $2 \mathrm{kHz}$ & $3 \mathrm{kHz}$ & $4 \mathrm{kHz}$ & $6 \mathrm{kHz}$ & $8 \mathrm{kHz}$ & & & \\
\hline$M$ & 69 & L & 0 & 15 & 25 & 55 & 65 & 75 & 70 & 70 & 80 & 39 & 4.1 & 2.5 \\
\hline $\mathrm{F}$ & 23 & $R$ & 0 & 25 & 50 & 60 & 100 & 110 & 105 & 105 & 105 & 123 & 2.1 & 1.6 \\
\hline$M$ & 81 & $R$ & 20 & 50 & 50 & 55 & 70 & 80 & 80 & 80 & 80 & 26 & 3.6 & 2.3 \\
\hline$M$ & 73 & L & 45 & 40 & 40 & 55 & 55 & 65 & 75 & 65 & 75 & 15 & 3.5 & 2.0 \\
\hline$M$ & 62 & $R$ & 45 & 40 & 50 & 55 & 60 & 65 & 70 & 85 & 90 & 154 & 3.3 & 2.1 \\
\hline$M$ & 20 & L & 20 & 30 & 40 & 45 & 55 & 70 & 70 & 85 & 75 & 98 & 3.7 & 2.3 \\
\hline $\mathrm{F}$ & 42 & $R$ & 50 & 45 & 40 & 40 & 55 & 85 & 110 & 90 & so & 182 & 3.5 & 2.0 \\
\hline M & 65 & L & 35 & 30 & 50 & 60 & 60 & 65 & 80 & 95 & 95 & 63 & 3.5 & 2.0 \\
\hline$M$ & 27 & $R$ & 20 & 20 & 25 & 35 & 100 & 105 & 105 & 120 & 110 & 17 & 3.5 & 2.0 \\
\hline$M$ & 76 & $R$ & 35 & 35 & 35 & 50 & 50 & 65 & 65 & 65 & 95 & 73 & 3.5 & 2.0 \\
\hline$M$ & 23 & $R$ & 5 & 10 & 5 & 25 & 100 & 105 & 105 & 105 & 95 & 24 & 3.5 & 2.0 \\
\hline$M$ & 73 & $R$ & 15 & 30 & 40 & 50 & 65 & 70 & 75 & 75 & 80 & 96 & 3.8 & 2.4 \\
\hline
\end{tabular}

M: male, F: female, R: right, L: left, HA: hearing aid, CR: compression ratio, SO: scale out 
SoundRecover for Phonak hearing aids and Sound Shaper for GN ReSound hearing aids. The NLFC parameters were determined individually using the manufacturer's software default settings based on the audiogram, In SoundRecover, the maximum audible output frequency was measured with frequency compression was deactivated. The input frequency corresponding to the maximum audible output frequency was then calculated using the equation from the study by Simpson, et al. [15]. The program recommended a setting that fit the bandwidth of compression within the individual' region of audibility using the highest cutoff frequency and lowest CR possible. In Sound Shaper, four predetermined adjustment modes, including off, mild, moderate, and strong, are included in the software in order to select the cut off frequency and CR. If the audiogram has a slope off $10 \mathrm{~dB}$ or greater and the slope begins at $2 \mathrm{kHz}$, a 'moderate' setting is recommended. In this study, all participants using GN Resound hearing aids, moderate NLFC setting was selected. The cut-off frequency ranged from $2.1 \mathrm{kHz}$ to $4.1 \mathrm{kHz}$, and the CR used in the current investigation was $1.6: 1$ to $2.5: 1$. Other additional hearing aid features were disabled during the trial period. Because the purpose of the study was to investigate the effects of NLFC, and because the interactions between advanced features and NLFC are not yet known, features except for NLFC, such as noise reduction, were de-activated during testing.

\section{Test battery administration}

All participants were tested under three conditions: unaided, NLFC-off, and NLFC-on, and had a two-week acclimatization period between each condition. Participants were blinded to the hearing-aid setting condition. The order of test and hearing-aid setting was counterbalanced. Performance was repeatedly evaluated with consonant tests in quiet and noise settings, speech perception in noise, music perception, and acceptableness of sound quality rating tasks. In addition, two questionnaires, the Abbreviated Profile of Hearing Aid Benefit (APHAB) and International Outcome Inventory-Hearing Aids (IOI-HA), were assessed.

\section{Speech perception}

Speech perception testing involved the consonant recognition test and the speech perception test in 4-talker babble noise. For the consonant recognition test [16], participants sat in a chair in the center of the sound field towards two loudspeakers located approximately one meter away from the front $\left(0^{\circ}\right)$. The consonant test was conducted in quiet and steady noise [0 and $5 \mathrm{~dB}$ signal to noise (SNR)] conditions. The target stimuli consisted of 18 Korean bisyllablic nonsense words (/a/ - target consonant + /a/, i.e., /a-na/ or /a-sa/) that had been recorded with a male speaker. Target consonant and noise were presented at a calibrated level of $65 \mathrm{~dB}$ SPL. Each of the 18 target words was randomly presented three times. Participants were instructed to select the target word from the 18 words displayed on the screen. The number of correct answers was divided by a total 54 target words and multiplied by 100 to calculate the percentage of correct answers (\%). Before the experiment, four to five practice tests were conducted. No feedback was provided.

The speech perception was also evaluated by determining the percentage of correct responses in the open set word recognition and sentence recognition using Korean Standard Sentence Lists for Adults [17]. Testing was performed with background babble noise at $5 \mathrm{~dB}$ SNR. Forty recorded words and 10 recorded sentences were presented one meter away from the patient. All of the words and sentences were selected at random to reduce error by sequence.

\section{Music perception}

Music perception was assessed by the Korean version of the Clinical Assessment of Music Perception test (K-CAMP) [18]. For the K-CAMP test, a custom MATLAB ${ }^{\circledR}$ (MathWorks, Inc., Natick, MA, USA) program was used to present stimuli on a laptop computer. A single loud-speaker that presented the target stimuli was positioned one meter from the participant. Two subtests were included in the protocol: pitch direction discrimination and timbre identification. The complex-tone pitch direction discrimination test was performed using a oneup, one-down procedure and two-alternative forced-choice using three base frequencies $(262,330$, and $391 \mathrm{~Hz})$. The tones were presented at $65 \mathrm{dBA}$ and lasted $760 \mathrm{~ms}$. The test started with a 12-semitone interval and the step size was one semitone. Each patient was asked to select the higher pitch using two buttons on a computer screen. The just-noticeable difference limen (DL) in semitones was calculated with the mean interval size for three adaptive tracks.

The timbre identification test consisted of 24 presentations of recorded sounds of eight musical instruments: piano, violin, cello, acoustic guitar, trumpet, flute, clarinet, and saxophone. Each sound clip was played three times in random order at $65 \mathrm{dBA}$. The percentage of correct answers was analyzed after 24 presentations.

\section{Sound quality rating}

The speech and music sound quality were rated using a visual analogue scale, where 0 represented being not at all acceptable and 10 indicated very acceptable. The sound quality of music for piano, string instruments, and classical music was evaluated based on the following five aspects: loudness, richness, clarity, natural sound and sharpness. In addition, sound 
quality on speech in noise background was also evaluated for clarity, distortion, sharpness, and additive noise.

\section{Questionnaires}

All participants were asked to complete an outcome questionnaire that assessed hearing aid benefits. Daily listening and communication abilities were investigated using an APHAB questionnaire, a 24-question of four aspects of auditory function: communication difficulty, effect of background noise, effect of reverberation, and aversiveness to loud sounds. The IOI-HA is a self-assessment inventory that estimates multiple outcome domains, such as use, benefit, satisfaction, residual activity limitations, residual participation restrictions, impact on others, and quality of life. A score of $1-5$ was assigned to each of the seven questions, with higher scores reflective of more positive outcomes. The Korean versions of the APHAB [19] and IOI-HA [20] questionnaires have been properly translated and validated, and are used as reliable and valid tools for assessment of self-reported outcomes in South Korea. Finally, we also investigated the overall satisfaction using a visual analogue scale; the purchase intention and recommendation intention for hearing aids with frequency lowering technology were also assessed.

\section{Statistical analysis}

All results were analyzed using SAS, version 9.4 (SAS Institute Inc., Cary, NC, USA). To compare speech perception, music perception, and subjective assessments between the three listening conditions (unaided, NLFC-on, and NLFC-off), repeated measures one-way analysis of variance or the Friedman test were conducted depending on the test of normality. If there were significant differences among the three listening condi- tions, post-hoc analysis using Bonferroni correction and Wilcoxon signed-rank test was conducted to analyze differences among the different listening conditions. A paired t-test was conducted to compare the overall satisfaction and K-IOI-HA results between the NLFC-on and NLFC-off conditions.

\section{Ethics statement}

All tests were conducted with the approval of the Institutional Review Board of the Samsung Medical Center (IRB no. 2016-03-017).

\section{Results}

\section{Speech perception}

The average pure-tone thresholds among listening conditions are shown in Fig. 1B. There was a significant difference in pure-tone thresholds between unaided and aided conditions at frequencies above $1 \mathrm{kHz}$ (all $p<0.001$ ). However, the puretone threshold was similar between the two aided listening conditions (NLFC-on and -off).

Fig. 2A shows the results of consonant perception in quiet and noisy backgrounds. A significant difference was observed in consonant perception under quiet conditions when comparing the NLFC-off ( $p=0.0002)$ and NLFC-on ( $p=0.0075)$ statuses to unaided conditions, but not between NLFC-on and -off settings. In a steady noise situation (SNR $0 \mathrm{~dB}$ ), consonant perception increased significantly when tested in the NLFC-on state compared with the unaided state $(p=0.0198)$. In addition, consonant perception also increased significantly in the NLFC-off state compared to the unaided condition in a steady noise (SNR $5 \mathrm{~dB})(p=0.0125)$. However, there was no significant difference in consonant perception between the
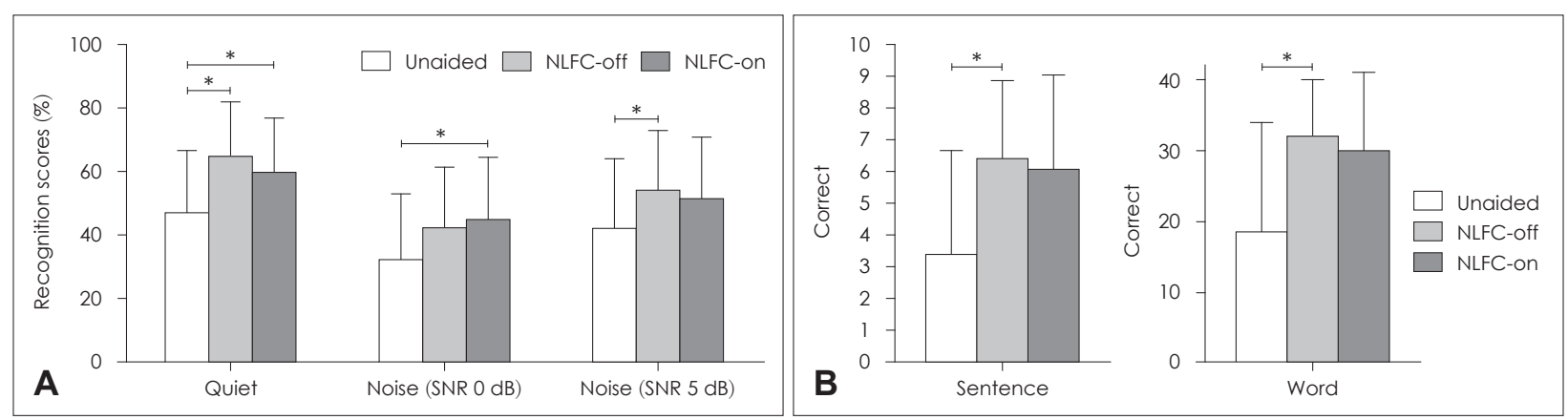

Fig. 2. Consonant and speech perception. A: Consonant perception in quiet and noise backgrounds. A significant difference was observed in consonant perception under quiet conditions when the NLFC-off $(p=0.0002)$ and NLFC-on $(p=0.0075)$ conditions were compared with the unaided condition, but not between the NLFC-on and -off conditions. In a steady noise situation (SNR $0 \mathrm{~dB}$ ), consonant perception increased significantly when tested in the NLFC-on condition over the unaided condition $(p=0.0198)$. In addition, consonant perception also increased significantly in the NLFC-off state compared to the unaided condition in a steady noise situation (SNR $5 \mathrm{~dB}$ ) $(p=0.0125)$. However, there was no significant difference in consonant perception between the NLFC-on and -off states in both quiet and noisy backgrounds. B: Speech perception in noise conditions. Speech perception with sentences and words was significantly increased when tested in the NLFC-off over the unaided condition ( $p=0.0156$ for sentence recognition, $p=0.0066$ for word recognition). No significant difference was identified in speech perception between the NFLC-on and -off conditions. NLFC: non-linear frequency compression, SNR: signal to noise. 


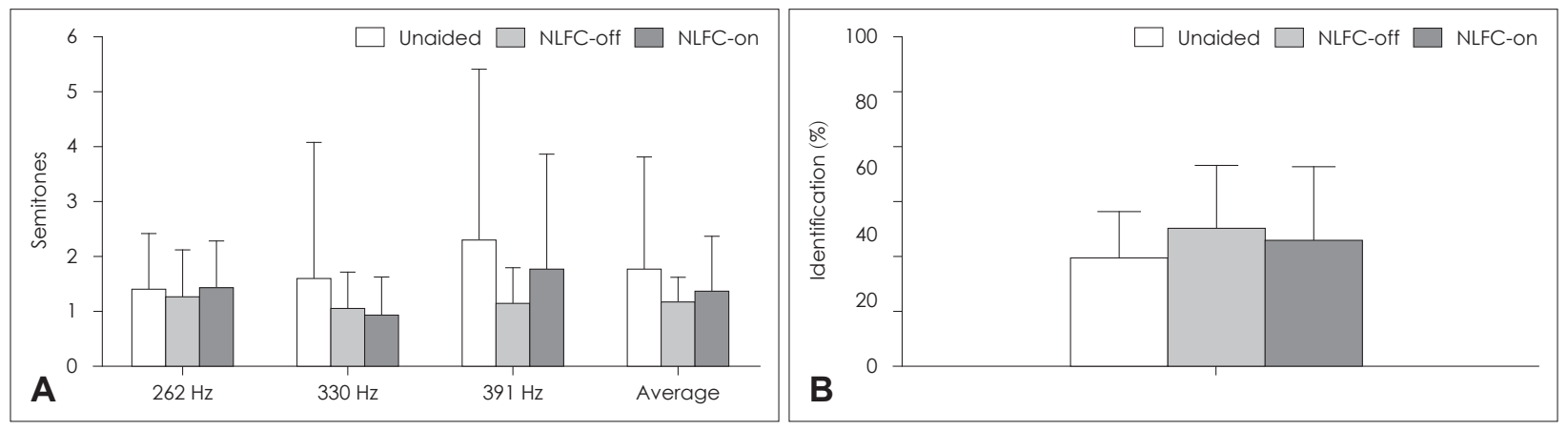

Fig. 3. Music perception. A: Pitch differentiation scores at the three fundamental frequencies were not meaningfully improved among three different listening situations (unaided, NLFC-on and -off). B: The difference in the average timbre identification score among the three conditions was not significant. NLFC: non-linear frequency compression.

NLFC-on and -off conditions in both quiet and noisy backgrounds.

The speech perception in babble noise was compared at 5 $\mathrm{dB}$ SNR, and the speech perception in the three different listening conditions is presented in Fig. 2B. Post-hoc analysis determined that speech perception using sentences and words was significantly increased when tested in the NLFC-off compared with the unaided condition ( $p=0.0156$ for sentence recognition, $p=0.0066$ for word recognition). No significant difference in speech perception between the NFLC-on and -off conditions was noted.

\section{Music perception}

\section{Pitch direction discrimination tests}

DLs in an unaided condition were $1.42 \pm 1.01,1.6 \pm 2.48$, and $2.3 \pm 3.12$ semitones at base frequencies of 262,330, and $391 \mathrm{~Hz}$, respectively. As the base frequency increased, DLs became wider. At base frequencies of 262, 330, and $391 \mathrm{~Hz}$, DLs were $1.27 \pm 0.85,1.07 \pm 0.66$, and $1.16 \pm 0.64$ semitones in the NLFC-off condition, and $1.42 \pm 0.87,0.93 \pm 0.71$, and $1.77 \pm 2.11$ in the NLFC-on condition (Fig. 3A). Overall, pitch discrimination scores at the three base frequencies were not significantly improved among the three different listening situations (unaided, NLFC-on, and -off).

\section{Timbre identification}

The mean identification of the musical instrument sound was $34.38 \pm 14.23 \%$ for the unaided condition, $43.75 \pm 19.9 \%$ for the NLFC-off condition, and $39.93 \pm 22.99 \%$ for the NLFCon condition. The difference in the average timbre identification score among the three conditions was not significant (Fig. 3B).

\section{Sound quality rating}

Fig. 4 displays the averaged speech ratings in noise condi-

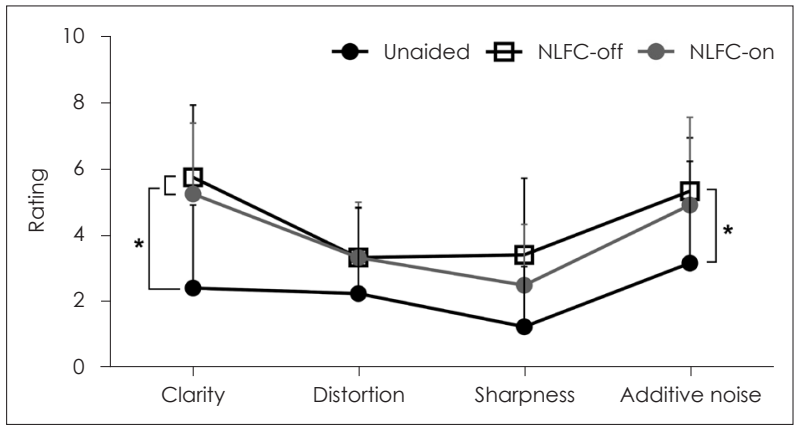

Fig. 4. Sound quality for speech. Satisfaction of sound quality was increased in the NLFC-on and -off conditions compared to unaided conditions in terms of clarity ( $p=0.0018$ for NLFC-off, $p=0.0075$ for NLFC-on). However, there was no difference between ratings with NLFC and without NLFC. NLFC: non-linear frequency compression.

tions. The results showed increased satisfaction of sound quality in NLFC-on and -off conditions compared to the unaided conditions in terms of clarity ( $p=0.0018$ for NLFC-off, $p=0.0075$ for NLFC-on). In regard to noise reduction, subjective satisfaction was remarkably increased in the NLFC-off condition, compared with the unaided condition $(p=0.0149)$. However, there was no difference between ratings with NLFC and without NLFC.

For the musical quality assessment, participants reported a more satisfying sound quality of piano with NLFC-on than with the unaided condition for loudness $(p=0.0195)$ and clarity ( $p=0.0369$ ). In addition, the clarity of stringed instruments was significantly improved in the NLFC-off condition compared to the unaided condition ( $p=0.0093$ ). However, no improvement in the musical quality perceived through NLFC compared to without NLFC was found.

\section{Questionnaires}

Subjective hearing disability was measured using the APHAB and K-IOI-HA. For APHAB (Fig. 5A), participants reported decreased hearing handicap for the properties of ease of com- 
munication, reverberation, and background noise with both NLFC-on and -off conditions, compared to the unaided condition. Compared to the unaided state, significant improvement was found in ease of communication $(p=0.0006)$ and background noise ( $p=0.0138)$ in the NLFC-off condition. In addition, participants reported increased ease of communication in the NLFC-on than in the unaided condition $(p=0.0034)$. However, there was no difference in subjective improvement in hearing disability between NLFC-on and -off conditions. For K-IOI-HA, the mean score was $24.42 \pm 3.63$ and $22.67 \pm 4.94$ in the NLFC-off and -on conditions, respectively (Fig. 5B); however no significant improvements were identified.

Based on the overall satisfaction using a visual analogue scale, of the twelve participants, five reported increased satisfaction with the use of the NLFC function, six reported decreased, and one reported no change in satisfaction. The average score in the NLFC-on and -off conditions was $4.90 \pm 2.23$ and 5.34 \pm 2.60 , respectively. Rather, the average overall satisfaction was high in the condition without NLFC, but the result was not statistically significant. In the NLFC-off condition, six patients $(50 \%)$ reported that they were willing to purchase a hearing aid, and seven (58.33\%) said they would like to recommend it to others. For the NLFC-on condition, purchase and recommendation intentions were found in three $(25 \%)$ and seven $(58.33 \%)$ patients, respectively.

\section{Discussion}

The aim of this study was to assess the influence of NLFC on several performance outcomes including consonant perception in quiet and noise, speech perception in noise, music perception, and acceptableness of sound quality for Korean adult listeners with high-frequency hearing loss. The NLFC effect was established by comparing performance with three different listening conditions: unaided, NLFC-on and -off. The results did not show a significant positive effect of activating NLFC on speech and music perception or for sound quality.

Previous studies have produced inconsistent results on the influence of NLFC on consonant and speech perception in hearing-impaired listeners. McCreery, et al. [5] inspected word recognition in hearing impaired children and adults with NLFC or CP. The results showed higher recognition performance with NLFC than CP in both children and adults. In addition, a significant improvement in the average perception rate of fricatives and affricates due to NLFC activation was confirmed in a study by Alexander, et al. [6]. These results indicate that the NLFC was effective at improving high-frequency audibility on average, resulting in improvements in high-frequency speech sound recognition.

Although there have been positive findings in some of the previous studies, this study found no significant difference between the NLFC-on and -off conditions. According to the longterm average speech spectrum (LTASS) analysis, individual languages exhibit many significant variations, differing up to $3 \mathrm{~dB}$ in magnitude from the average value [21,22]. The differences in the distribution of phonemes among the different languages might contribute to the variation in LTASS. The results of the Korean LTASS revealed significantly lower levels in frequencies above $2 \mathrm{kHz}$, compared to English [23]. Compared with English, the lower frequency distribution of highfrequency phonemes in Korean might contribute to the lower levels of the LTASS in high-frequency areas [24]. Therefore, it is hypothesized that the improvement of high-frequency audibility through NLFC would have limited benefit to Korean listeners' consonant and speech perception due to this difference in LTASS and phoneme distribution.

There are currently only a limited number of studies that have focused on the efficacy of NLFC at the sentence level.
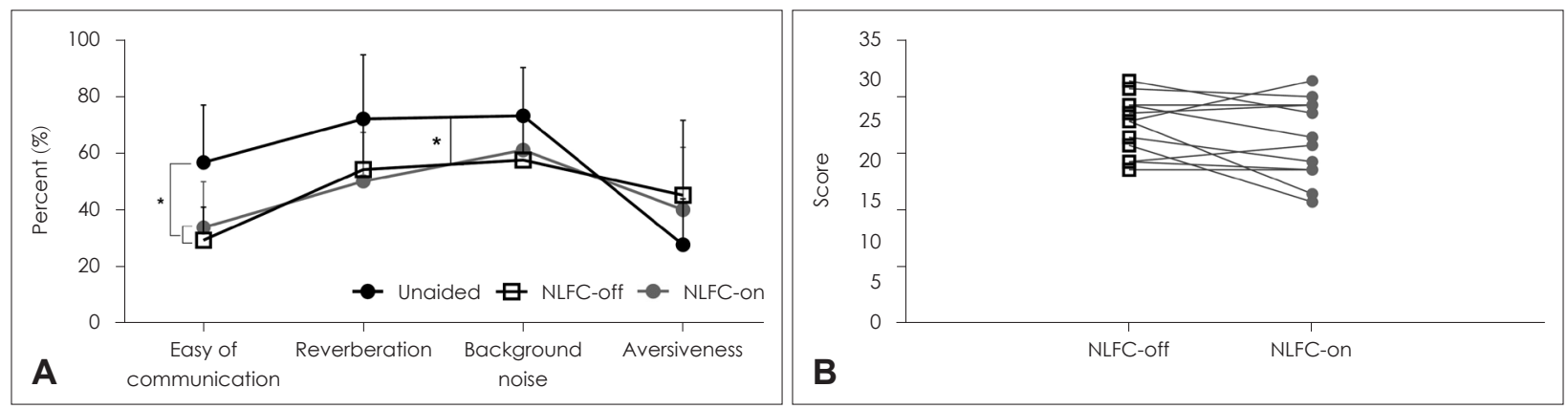

Fig. 5. Hearing disability. A: For Abbreviated Profile of Hearing Aid Benefit, participants reported decreased hearing handicaps in ease of communication, reverberation, and background noise with both NLFC-on and -off conditions compared to the unaided. Compared to the unaided condition, significant improvement was found in ease of communication $(p=0.0006)$ and background noise $(p=0.0138)$ in the NLFC-off condition. In addition, participants reported increased ease of communication in the NLFC-on, compared with the unaided condition $(p=0.0034)$. However, there was no difference in subjective improvement in hearing disability between NLFC-on and -off conditions. B: For Korean version of the International Outcome Inventory-Hearing Aids, no significant improvement was found. NLFC: nonlinear frequency compression. 
Simpson, et al. [10] studied sentence perception in eight talker babble maskers for five hearing-impaired adults with sloping high-frequency hearing loss. Only one out of five subjects showed a meaningfully lower speech reception threshold with NLFC than with CP. Other than the consonant recognition, speech perception at the sentence level is a more complex task. Compared to recognition of consonant and vowels, sentence recognition requires listeners' prior linguistic knowledge and higher-level cognitive processes. Therefore, the use of hearing aids with NFLC function may not dramatically influence their recognition accuracy of speech sentences.

The importance of music appreciation is a highly valuable component of the quality of life. Uys, et al. [11] achieved a comprehensive investigation of the effects of NLFC on music perception by using a Music Perception Test in 40 adults hearing aid users who had moderate to severe hearing loss. The results indicated that timbre and melody improved with NLFC compared to without NLFC. However, we could not confirm the positive effects of NLFC on music perception in this study. Since NLFC might potentially distort the harmonic ratio in the original sound, it is thought to have negative effects on music perception. Although there was no statistical significance, the results of this study indicated that the mean pitch discrimination and timbre identification were better in the NLFC-off condition than in the NLFC-on. Besides, considering that the sound quality and overall satisfaction with NLFC did not improve compared to the cases without NLFC, it is possible that excessive compression occurred.

Our study had several limitations. First, the presence of CDR was not confirmed. Since the presence of CDR was not considered in the NLFC setting process, it may be difficult to expect the benefit of the NLFC technique if the destination band falls within the dead region. Second, real-ear probe microphone measures were not performed when fitting NLFC amplification. The fact that the audibility of reduced-frequency speech sounds was not confirmed may have influenced the unfavorable results of this study. Third, sufficient acclimatization period was not considered. Auditory acclimatization plays an important role in speech perception. Some studies recommended an adaptation period of NLFC for several weeks before actual testing to help participants become familiar with the tested technique $[4,25,26]$. These studies indicated that acclimatization period for NLFC fitting may not be beneficial for every hearing-impaired listener. However, a relatively longer time for the acclimatization period seems to improve at least some hearing-impaired listeners' perceptual performance. Although previous studies presented various results with respect to the acclimatization effect across listeners, it is possible that the two-week adaptation period in this study was insufficient to demonstrate a significant effect of NLFC.

In conclusion, for Korean hearing aid users with high-frequency hearing loss, activating NLFC did not improve consonant and speech perception both in quiet and noise conditions, compared with the NLFC-off condition. In addition, activating NLFC did not affect sound quality as well as music perception, compared to $\mathrm{CP}$. These results suggest that activating NLFC may have limited clinical benefit, even if the candidate has extremely high-frequency hearing loss. Thus, clinicians should be thoughtful about applying NLFC. Specifically, language characteristics, fine fitting with the appropriate individualized NLFCs setting must be considered to maximize audibility of high-frequency components, while minimizing the detrimental effects of spectral distortion in this technology.

\section{Acknowledgments}

We would like to thank all subjects who participated in this study and our colleagues at the Hearing Research Lab in Samsung Medical Center for performing the auditory tests.

\section{Conflicts of interest}

The authors have no financial conflicts of interest.

\section{Author Contributions}

Conceptualization: Il Joon Moon. Data curation: Jungmin Ahn and Ju Yong Kang. Formal analysis: Jungmin Ahn. Methodology: Ji Eun Choi. Project administration: Il Joon Moon, Ik Joon Choi, Myung-Chul Lee, and Byeong-Cheol Lee. Visualization: Jungmin Ahn. Writing — original draft: Jungmin Ahn. Writing — review \& editing: Jungmin Ahn, Sung Hwa Hong, and Il Joon Moon. Approval of final manuscript: all authors.

\section{ORCID iDs}

Jungmin Ahn

Ji Eun Choi

Ju Yong Kang

Ik Joon Choi

Myung-Chul Lee

Byeong-Cheol Lee

Sung Hwa Hong

Il Joon Moon

https://orcid.org/0000-0003-2029-6243 https://orcid.org/0000-0001-8105-813X https://orcid.org/0000-0002-6700-5629 https://orcid.org/0000-0002-9680-3873 https://orcid.org/0000-0002-2574-4976 https://orcid.org/0000-0002-4149-273X https://orcid.org/0000-0001-6906-8925 https://orcid.org/0000-0002-3613-0734

\section{REFERENCES}

1) Chang YS, Yoon SH, Kim JR, Baek SY, Cho YS, Hong SH, et al. Standard audiograms for Koreans derived through hierarchical clustering using data from the Korean National Health and Nutrition Examination Survey 2009-2012. Sci Rep 2019;9:3675.

2) Stelmachowicz PG, Pittman AL, Hoover BM, Lewis DE. Effect of stimulus bandwidth on the perception of $/ \mathrm{s} /$ in normal- and hearingimpaired children and adults. J Acoust Soc Am 2001;110:2183-90.

3) Pepler A, Munro KJ, Lewis K, Kluk K. Prevalence of cochlear dead regions in new referrals and existing adult hearing aid users. Ear Hear 2014;35:e99-109.

4) Wolfe J, John A, Schafer E, Nyffeler M, Boretzki M, Caraway T. Evaluation of nonlinear frequency compression for school-age children with moderate to moderately severe hearing loss. J Am Acad Audiol 2010;21:618-28. 
5) McCreery RW, Alexander J, Brennan MA, Hoover B, Kopun J, Stelmachowicz PG. The influence of audibility on speech recognition with nonlinear frequency compression for children and adults with hearing loss. Ear Hear 2014;35:440-7.

6) Alexander JM, Kopun JG, Stelmachowicz PG. Effects of frequency compression and frequency transposition on fricative and affricate perception in listeners with normal hearing and mild to moderate hearing loss. Ear Hear 2014;35:519-32.

7) Glista D, Scollie S, Bagatto M, Seewald R, Parsa V, Johnson A. Evaluation of nonlinear frequency compression: clinical outcomes. Int J Audiol 2009;48:632-44.

8) Perreau AE, Bentler RA, Tyler RS. The contribution of a frequency-compression hearing aid to contralateral cochlear implant performance. J Am Acad Audiol 2013;24:105-20.

9) Sakamoto S, Goto K, Tateno M, Kaga K. Frequency compression hearing aid for severe-to-profound hearing impairments. Auris Nasus Larynx 2000;27:327-34.

10) Simpson A, Hersbach AA, McDermott HJ. Frequency-compression outcomes in listeners with steeply sloping audiograms. Int J Audiol 2006;45:619-29.

11) Uys M, Pottas L, Vinck B, van Dijk C. The influence of non-linear frequency compression on the perception of music by adults with a moderate to sever hearing loss: subjective impressions. S Afr J Commun Disord 2012;59:53-67.

12) Parsa V, Scollie $S$, Glista $D$, Seelisch $A$. Nonlinear frequency compression: effects on sound quality ratings of speech and music. Trends Amplif 2013;17:54-68.

13) Bohnert A, Nyffeler M, Keilmann A. Advantages of a non-linear frequency compression algorithm in noise. Eur Arch Otorhinolaryngol 2010;267:1045-53.

14) Picou EM, Marcrum SC, Ricketts TA. Evaluation of the effects of nonlinear frequency compression on speech recognition and sound quality for adults with mild to moderate hearing loss. Int J Audiol 2015;54:162-9.

15) Simpson A, Hersbach AA, McDermott HJ. Improvements in speech perception with an experimental nonlinear frequency compression hearing device. Int J Audiol 2005;44:281-92.
16) Chang SA, Park MH, Oh SH, Lee KM. Influence of upper frequency boundary on consonant perception in normal hearing and cochlear implant adults. Audiol Speech Res 2017;13:231-9.

17) Jang H, Lee J, Lim D, Lee K, Jeon A, Jung E. Development of Korean standard sentence lists for sentence recognition tests. Audiology 2008;4:161-77.

18) Jung KH, Cho YS, Cho JK, Park GY, Kim EY, Hong SH, et al. Clinical assessment of music perception in Korean cochlear implant listeners. Acta Otolaryngol 2010;130:716-23.

19) Lim HJ, Park MK, Cho YS, Han GC, Choi JW, An YH, et al. Validation of the Korean version of the abbreviated profile of hearing aid benefit. Korean J Otorhinolaryngol-Head Neck Surg 2017;60: 164-73.

20) Chu H, Cho YS, Park SN, Byun JY, Shin JE, Han GC, et al. Standardization for a Korean adaptation of the international outcome inventory for hearing aids: study of validity and reliability. Korean J Otorhinolaryngol-Head Neck Surg 2012;55:20-5.

21) Byrne D. The speech spectrum--some aspects of its significance for hearing aid selection and evaluation. Br J Audiol 1977;11:40-6.

22) Byrne D, Dillon H. The National Acoustic Laboratories' (NAL) new procedure for selecting the gain and frequency response of a hearing aid. Ear Hear 1986;7:257-65.

23) Noh H, Lee DH. Cross-language identification of long-term average speech spectra in Korean and English: toward a better understanding of the quantitative difference between two languages. Ear Hear 2012;33:441-3.

24) Byun SW. Frequencies of Korean syllables and the distribution of syllables of PB word list. Korean J Otolaryngol-Head Neck Surg 2003;46:737-41.

25) John A, Wolfe J, Scollie S, Schafer E, Hudson M, Woods W, et al. Evaluation of wideband frequency responses and nonlinear frequency compression for children with cookie-bite audiometric configurations. J Am Acad Audiol 2014;25:1022-33.

26) Glista D, Scollie S, Sulkers J. Perceptual acclimatization post nonlinear frequency compression hearing aid fitting in older children. J Speech Lang Hear Res 2012;55:1765-87. 